\title{
XVI.
}

\section{Ein neuer Fall von Hermaphroditismus (H. spurius masculinus?),}

\author{
an Lebenden beobachtet, mitgetheilt \\ von Prof. Dr. Marchand in Giessen. \\ (Hierzu Taf. IX.)
}

Das Individuum, welches die in der Ueberschrift bezeichnete Anomalie darbietet, Marie Raab, ist geboren zu Callbach, Kreis Büdingen, Grossherzogthum Hessen, am 20. August 1853, also zur Zeit der Untersuchung $29 \frac{1}{2}$ Jahre alt.

Ihre Eltern - wir bezeichnen die Raab vorläufig, ihrem bisherigen Personenstande entsprechend, als weiblich - sind nicht mehr am Leben. Der Vater starb, als sie 18 Jahre alt war, die Mutter 3 Jahre früher. Sie lebt jetzt bei ihrem einzigen älteren Bruder, für welchen sie Haus- und Feldarbeit ausführt, und hat sich bisher stets weiblich gekleidet. Seit dem 16. oder 17. Jahre hat sich indess bei dieser Person eine sehr ausgesprochene Neigung zum weiblichen Geschlechte bemerklich gemacht, von welcher Andeutungen wohl auch früher in ihrer Schulzeit schon vorhanden gewesen zu sein scheinen, so dass ihr damals bereits die Eltern, welche von der Missbildung ihrer Genitalien selbstverständlich Kenntniss hatten, mehrfach den Verkehr mit Mädchen verboten. Seit der Pubertät hat die Marie Raab indess eingestandenermaassen häufig den Coitus mit weiblichen Personen ausgetibt, und zwar, wie aus ihren Angaben hervorgeht, mit recht gutem Erfolge. Auch spontane Entleerungen einer weisslichen Flüssigkeit aus den Genitalien will sie nicht selten gehabt haben, dagegen bestreitet sie ausdrücklich, dass jemals Blutabgang stattgefunder habe.

Der abnorme Zustand ihrer Genitalverhältnisse ist in ihrem Heimathsort wohlbekannt, und ist, wie es scheint, die Veranlassung gewesen, dass die Person wegen angeblicher geistiger Be- 
Iircharo's Archio. Bd. XCII.

1.

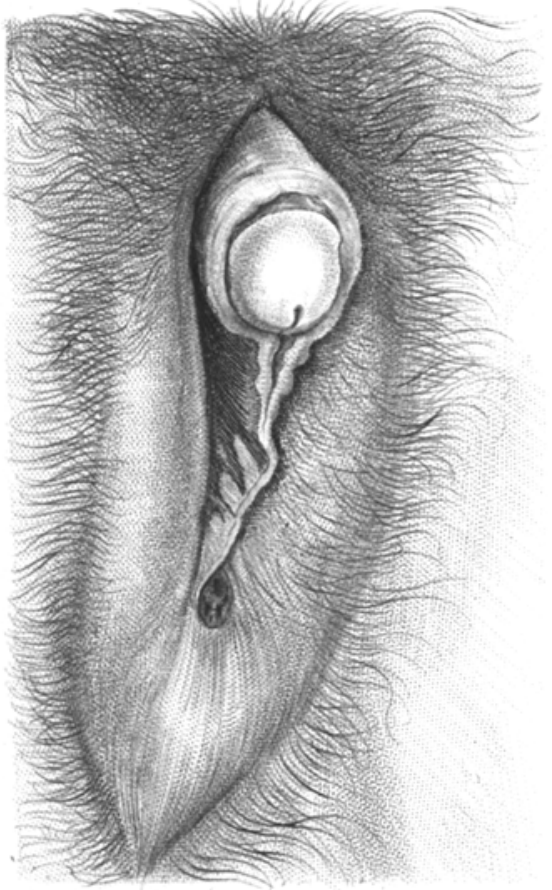

Tet. $R$.

2

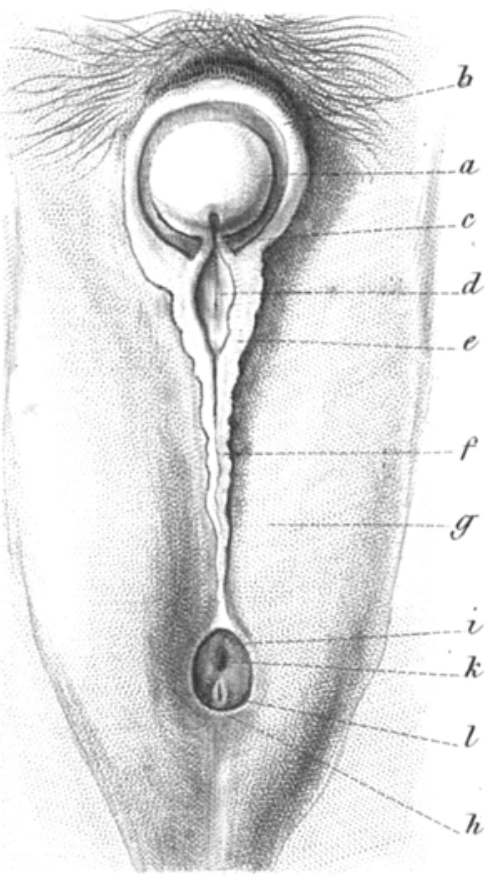

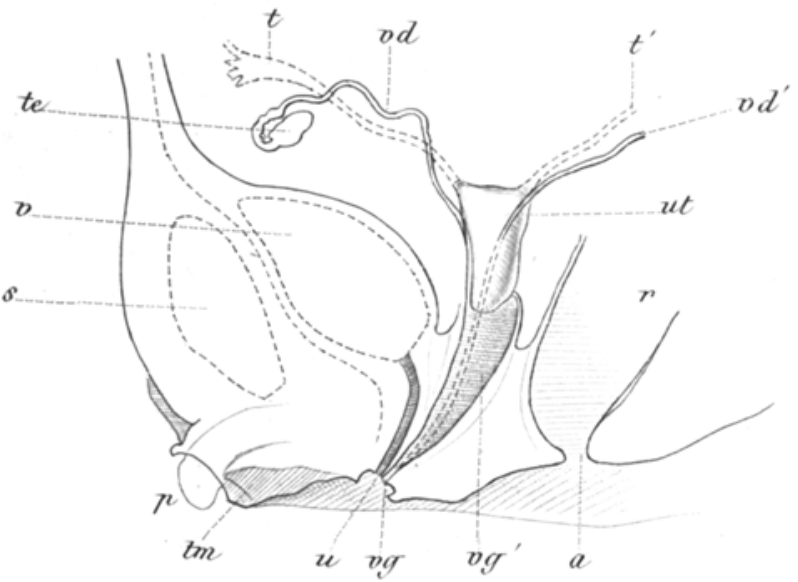

WT Gromann so. 
schränktheit, welche jedoch thatsächlich nicht erheblich ist, unter Curatel gestellt wurde. Bei Gelegenheit einer ihrer Klagen über Misshandlungen Seitens ihres Bruders, welcher ihre Abhängigkeit zu missbrauchen scheint, hatte Herr Kreisarzt Dr. Brettel in Büdingen Veranlassung, die Person zu untersuchen, und wir verdanken der Aufmerksamkeit und dem Interesse dieses Herrn die Gelegenheit, die M. Raab hier in Giessen einer genauen Untersuchung zu unterwerfen, was um so leichter zu erreichen war, als die bedauernswerthe Person lebhaft den Wunsch hat, als Mann anerkannt zu werden, um sich der Beschränkung ihrer persönlichen Freiheit entziehen zu können.

Die Untersuchung wurde am 14. Februar 1883 vom Verf. gemeinsam mit Prof. Ahlfeld, dessen geübter Hand hauptsächlich das Resultat der inneren Untersuchung zu danken ist, und Dr. Brettel in der hiesigen medicinischen Klinik, wo die Person Aufnahme gefunden hatte, vorgenommen. Am 15. d. M. wurde dieselbe der hiesigen medicinischen Gesellschaft vorgestellt.

Marie Raab ist $158 \mathrm{~cm}$ gross, ziemlich schlank gebaut, und macht in ihrer weiblichen Kleidung auf den ersten Blick ganz den Eindruck eines weiblichen Individuums. Die Haare sind hellblond, in Zöpfe geflochten, welche bis über die Schulterblätter herabhängen. Die Kopf- und Gesichtsbildung ist indess eher männlich als. weiblich zu nemnen, die Stirn ziemlich hoeh, mit stark vorspringender Tubera frontalia, die Augenbrauen wenig geschwungen, die Nase gerade, der Mund ziemlich gross; Backen, Oberlippe und Kinn sind aber vollständig bartlos, nur mit zartem Flaum bedeckt; das Gesicht sieht aus, wie das eines 18jährigen Jünglings. Die Ohrläppchen sind angewachsen, von Ohrlöehern durchbohrt.

Der Kehlk opf springt deutlich, jedoch nicht besonders stark vor; die Stimme ist tief, aber nicht rauh. Die Raab giebt an, dass der Stimmwechsel im 17. Jahre erfolgt sei.

Der Thorax ist im oberen Theil flach gewölbt, und wird beim tiefen Athmen nur wenig gehoben, die Athmung ist mehr abdominal; das Athmungsgeräusch ist links rein vesiculär, rechts oben mit etwas verlängerter, rauher Exspiration. Der Umfang der Brust in der Höhe der Brustwarzen beträgt $81-86 \mathrm{~cm}$.

Die Mammae sind vollständig weiblich entwickelt, hängend, die Brustwarzen stark vorragend, mit wenig pigmentirtem, etwas höckerigem Warzenhof; durch das lockere, nicht besonders reichliche Fettpolster ist der stark gelappte, umfangreiche Drüsenkörper sehr deutlich durchzufühlen. Flüssigkeit lässt sich aus. der. Warze nicht hervorpressen. - Die Haut ist im Ganzen zart. Doch sind die Formen des Körpers nicht besonders weich und rundlich, sie erinnern vielmehr durch die stärker ausgeprägte Muscu- 
latur eher an den männlichen, als den weiblichen T'ypus. Dagegen ist die Taille schlauk, und die Hüften breit, so dass Bauch und Beckengegend einen entschieden weiblichen Eindruck machen, nur die Conjugata ext. ist kürzer als beim Weibe. Die Maasse des Beckens sind folgende:

Grösster Abstand der Spinae anter. sup. (aussen gemessen) 26,75

Abstand der Cristae . . . . . . . . . . . . . 27,5

Abstand der Trochantern. . . . . . . . . . 31,0

Länge der Conjugata ext. . . . . . . . . . . . 16,75,

Die äbrigen Hauptmaasse des Körpers sind:

Schulterbreite (über den Rücken gemessen) . . $42 \mathrm{~cm}$

Umfang der Taille . . . . . . . . . . . 68 .

Umfang des Beckens in der Höhe der Spina ant. sup. 80 -

Unfang um die Trochantern . . . . . . . . . . 96 -

Entfernung vom Nabel bis zur Symphyse . . . . 18 -

Entfernung vom Nabel bis zum Boden . . . . . . 96 -

Hände und Füsse sind ziemlich gross, die Arme lang; die Hände reichen bis über die Mitte der Oberschenkel hinab.

Die auffallendste Abweichung bieten num die Genitalien dar. Der Mons pubis ist wenig fettreich, mit starken blonden Haaren bedeckt, welehe sich nach oben scharf abgrenzen. Unterhalb des Schamberges tritt in der Mitte ein kleiner penisartiger Körper hervor, dessen Länge am Rücken yon der Wurzel bis zur Spitze $3 \mathrm{~cm}$ beträgt, die Dicke $1 \frac{1}{2} \mathrm{~cm}$.

Dieser Penis ist nach Angabe der M. R. der Erection fähig, wobei er über fingerlang und beträehtlich dicker werden soll. Der Penis trăgt eine anscheinend wohlgebildete, aber kleine Eichel, welche von dem Präputium nicht bedeckt ist; ihre Oberfläche ist hautartig, leicht gerunzelt. Der Penis ist jedoch nicht durchbohrt; an der Spitze der Eichel ist etwas nach unten, der Stelle der normalen Mündung entsprechend, eine flache Längsfurche sichtbar, deren Seitenränder nach abwärts in ein doppeltes kurzes Frenulum übergehen; das Frenulum tritt jederseits mit einer Hautfalte in Verbindung, welche die Fortsetzung des Präputium nach abwärts darstellt; zwisehen beiden Frenula bleibt in der Mitte eine längliche kahnförmige, mit Schleimhaut ausgekleidete Vertiefung, welche nach abwärts durch die spitzwinklig zusammentretenden, aus dem Präputium hervorgehenden Hautfalten abgeschlossen wird (Fig. 2 d, e). Durch die Vereinigung dieser letateren entsteht eine in der Medianlinie verlaufende, $3 \mathrm{~cm}$ lange und über $1 \mathrm{~cm}$ hohe Hautfalte, welche den Eindruck von zwei verschmolzenen kleinen Schamlippen macht. An diese allmählich niedriger werdende Raphe sehliesst sich nun eine rundliche, mit Schleimhaut ausgekleidete Vertiefung, eine Art Vestibulum an, deren unterer Rand durch ein halbmondförmiges Frenulum gebildet wird. Diese Yertiefung kann etwa die Spitze des Zeigefingers aufnehmen. Beim Auseinanderziehen der Ränder gewahrt man die Mündung der Harnröhre, durch welche man sehr leicht in der Richtung nach oben mit dem Katheter in die Blase gelangt. Di↔ Harnröhre verläuft etwas nach aufwärts gekrümmt; sie ist verhältnissmässig weit und $5 \mathrm{~cm}$ lang (wie 
sich durch Messung am Katheter beim Austritt der ersten Urintropfen ergiebt. Der Urin soll sich in starkem Strahl entleeren. Unmittelbar unterhalb der Harnröhrenmündung bemerkt man zwei sehr kleine Schleimhautfältchen, welche ein rudimentäres Hymen darzustellen scheinen, jedes etwa von der Grösse einer halben Linse, und zwischen diesen gelangt die Sonde in einen engen Gang, der anfangs etwas nach abwärts (resp. hinten), dann nach aufwärts in das Becken hinein führt, und $9 \mathrm{~cm}$ lang ist. Die Mündung dieses Ganges, welcher der Lage nach offenbar einer Vagina entspricht, lässt einen englischen Katheter No. 4 durchpassiren; weiter nach aufwärts hat die Sonde jedoch mehr Spielraum. Bei gleichzeitiger Einführung des Katheters und der Sonde in die Harnröhre und den Vaginalkanal liess sich zweifellos constatiren, dass beide Gänge zwar dicht hinter einander verlaufen, aber getrennt in dem Vestibulum münden. Die grossen Schamlippen, welche die beschriebezen Gebilde seitlich umgeben, und unterbalb des Yestibulum sich vereinigen, sind wenig umfang- und fettreich, schlaff, stark behaart. In denselben ist keine Spur eines festen Körpers, wie etwa eines Hodens oder Samenstranges fühlbar, auch an der Mändung des Leistenkanals findet sich nichts dergleichen. Die Länge des Perineu m beträgt $4,5 \mathrm{~cm}$.

Im Grossen und Ganzen machen die äusseren Genitalien somit mehr den Eindruck von rudimentär entwickelten weiblichen Organen mit ungewöhnlich stark entwickelter Clitoris und fast vollständiger Verschmelzung der kleinen Schamlippen.

Die in der Chloroformnarcose vorgenommene innere Untersuchung per anum ergab Folgendes:

Die in den Vaginalkanal eingeführte Sonde ist rom Rectum aus leicht durchzufühlen, und lässt sich im Becken hin- und herbewegen. Oberhalb ihres Endes gelangt der Finger an einen beweglichen Körper von rundlich walzenförmiger Gestalt, von etwa $4 \mathrm{~cm}$ Länge und Fingerdicke.

Das untere Ende dieses Körpers ist konisch, und scheint sich an den Vaginalkanal anzuschliessen nach Art einer Portio vaginalis, doch lässt sich eine directe Verbindung mit dem Vaginalkanal nicht constatiren. Das obere Ende dieses Körpers lässt sich, besonders bei starkem Herabdrängen desselben mit der anderen Hand, ebenfalls abtasten, und zwar kann man nach rechts oben eine Art Fortsatz, vielleicht ein Horn, fühlen. Bei Einführung des Katheters lässt sich erkennen, dass der untere Theil des beschriebenen Körpers wie die Portio vaginalis unter dem Blasenhals hervorragt. Die Lage des Körpers, welchen man für nichts anderes als einen Uterus halten kann, ist etwas schräg von rechts oben nach links unten geneigt.

Zur genaueren Feststellung des Verhaltens des Scheidenkanals wurde dieser nun auf Vorschlag des Prof. Ahlfeld durch eine Wasserinjection ausgedehnt. Dabei liess sich sofort vom Rectum aus der prall gefüllte ziemlich dünnwandige Schlauch hindurchfühlen, welcher nach oben allmählich sich bis zur Dicke eines Zeigefingers ausdehnte, und an seinem oberen 
Ende augenscheinlich den konischen Zapfen des präsumptiven. Uterus aufnahm, so dass die Natur des letzteren wohl zweifellos festgestellt war.

Rechts ron dem Uterus gelangt man mit dem Finger an einen sehr beweglichen rundlichen Körper, etwa von der Grösse einer kleinen plattgedrückten Kirsche; derselbe entschlüpt den Fingern sehr leicht; seine Lage entspricht etwa dem Leistenring; ob dieser Körper aber einen Hoden oder ein Ovarium darstellt, lässt sich nicht entscheiden.

Links ist zuweilen, ungefähr an der entsprechenden Stelle, ein weit kleineres ähnliches Körperchen zu fühlen.

Von einem Prostata-ähnlichen Körper ist keine Spur nachzuweisen, ebenso wenig von Samenblasen oder Ausführungsgängen.

Nach dem Ergebniss der inneren Untersuchung musste es somit noch zweifelhaft gelassen werden, ob hier Hermaphroditismus spurius männlichen oder weihlichen Geschlechtes vorliege, auch war die Möglichkeit eines wahren Hermaphroditismus keineswegs ausgeschlossen.

Das vollständige Fehlen einer menstruellen Blutung - sofern wir den Angaben der Person, welche zwar einen glaubwürdigen Eindruck macht, aber doch augenscheinlich für den Nachweis ihrer männlichen Natur interessirt ist, Glauben sehenken dürfen - würde an sich nicht als Beweis gegen einen wahren Hermaphroditismus oder gegen das Vorhandensein von Ovarien gelten können, denn die Menstruation fehlt auch bei notorisch weiblichem Geschlecht bei mehr oder weniger vollständiger Atrophie des Uterus und der Ovarien.

[Noch vor Kurzem hatten wir Gelegenheit die inneren Genitalien einer lange verheirathet gewesenen älteren Frau (aus der Praxis des Herrn Dr. Weber hierselbst) zu untersuchen, welche bei sehr enger rudimentärer Vagina einen sehr kleinen Uterus (ungefähr von der Grösse des Uterus eines 12 jährigen Mädchens) und vollständig, bis auf einen kleinen narbigen Streifen, geschwundene Ovarien besass, und welche niemals menstruirt gewesen war.]

Das gleichzeitige Vorhandensein von Hoden und Ovarien lässt sich durch die Untersuchung am Lebenden überhaupt nicht constatiren, und so lange dieser Nachweis fehlt, wird die Wahrscheinlichkeit stets ungleich mehr für die eine oder die audere Form des Pseudo-Hermaphroditismus sprechen, da die Fülle der ersteren Art überhaupt beim Menschen zu den allergrössten 
Seltenheiten gehören, wenn sie überhaupt als ganz zweifellos gelten können.

Von grosser Wichtigkeit für die Entscheidung wäre zunächst die Untersuchung des Secrets, welches nach den Angaben der Raab allérdings die Beschaffenheit von Sperma zu besitzen scheint, und sowohl beim Coitus als bei Pollutionen in ziemlich beträchtlicher Menge ejaculirt werden soll.

Während des 2tägigen Aufenthaltes der Raab in der Klinik. gelang es nicht, etwas von dem fraglichen Secret zu erhalten. Zwei Tröpfchen einer dünnen Flüssigkeit, welche uns noch dazu in eingetrocknetem Zustande übergeben wurden, enthielten keine charakteristischen Elemente. Indess muss bemerkt werden, dass auch das Fehlen von Spermatozoen in dem Secret selbstverständlich nicht gegen die männliche Natur der Geschlechtsdrüsen sprechen würde.

So lange wir über diesen Punkt keine Sicherheit besitzen, müssen wir uns selbstverständlich eines positiven Urtheils über die Natur der vorhandenen Geschlechtsdrüsen ganz enthalten. Wir können lediglich zu einem Wahrscheinlichkeitsresultate gelangen.

Fassen wir zu diesem Behufe die hauptsächlichen Befunde nochmals kurz zusammen: Wir haben eine Person unter Mittelgrösse vor uns, welche als Mädchen aufgewachsen ist, aber von früh auf sich selbst für ein männliches Individuum gehalten hat, ausgesprochen männliche geschlechtliche Neigungen besass, und auch diesen entsprechend handelte.

Der allgemeine Habitus des Körpers ist ein seltsames Gemisch von weiblichem and männlichem Typus, doch überwiegt der erstere augenscheinlich; männlich erscheinen die Gesichtsbildung, der Kehlkopf und die Stimme, die Formen der Musculatur, allenfalls noch die grossen Hände und Füsse; weiblich dagegen das lange Kopfhaar, die Bartlosigkeit, die stark ausgebildeten Brüste und der Bau des Körpers, namentlich der Bauchund Beckengegend. Die äusseren Genitalien ähneln ebenfalls weit mehr den weiblichen, als den männlichen: Kleiner undurchbohrter Penis, oder grosse Clitoris mit doppeltem Frenulum, unvollkommen getrennte, oder richtiger vereinigte kleine Schamlippen, sehr kleines Vestibulum 
mit getrennten Oeffnungen für Urethra und Vagina, keine Spur von Hoden in den seitlichen Hautfalten oder im Leistenkanal.

Die inneren Genitalien, soweit über dieselben Aufschluss erhalten werden kann, lassen einen $9 \mathrm{~cm}$ langen Vaginalkanal, einen ziemlich stark entwickelten Uterus, dagegen keine Spur von Prostata oder Samenblasen, und zwei seitlich gelagerte Körper erkennen, welche man mit gleichem Recht für Hoden oder Ovarien halten könnte.

Vergleichen wir den vorliegenden Befund mit dem, was über die anatomische Untersuchung derartiger Individuen mit "unsicheren Geschlechtsverhältnissen" bekannt ist, so finden wir im Grossen uud Ganzen eine Uebereinstimmung insofern, als es sich in der grossen Mehrzahl um männliche Individuen handelte, bei denen sowohl der Habitus des Körpers, als die Form der äusseren Genitalien und die Entwickelung der inneren Geschlechtskanäle mehr oder weniger deutlich dem weiblichen Typus entsprach.

Selten ist indess der allgemeine Habitus in so hohem Grade weiblich, wie bei der Raab, selbst die viel untersuchte und beschriebene Catharina Hohmann machte abgesehen von ihrer stark entwickelte Mammae mehr den Eindruck eines Mannes. (H. ist übrigens, wie ich von Prof. Ahlfeld erfahre, jetzt als Mann verheirathet, und soll sich sogar als Vater eines Kindes bekennen.)

Der Zustand der äusseren Genitalien ist von mehr untergeordneter Bedeutung; die bei der Raab vorhandene hypospadische Bildung ist insofern bemerkenswerth, als die beiden seitlichen, den Nymphen entsprechenden Hautfalten nur im oberen Theile getrennt, nach unten aber in einer länge von $3 \mathrm{~cm} z u$ einer einzigen medialen Hautfalte vereinigt sind, unterhalb deren sich das Vestibulum findet. Die den grossen Labien oder den Hälften des Scrotum entsprechenden Hautfalten sind schwächer entwickelt, als beim Weibe, sie umschliessen aber anscheinend keinen Processus vaginalis, jedenfalls keinen Hoden; es ist also, im Falle des Vorhandseins der letateren, vollständiger Cryptorchidismus da. Besonders bemerkenswerth ist nun die Existenz zweier getrennter Oeffnungen für die Urethra und den Genitalkanal, während in der Regel wenigstens ein kurzer Siuus uro- 
genitalis vorhanden ist, der hier nur durch das kleine Vestibulum gebildet wird. Aber auch dafür fehlt es nicht an Beispielen.

Unsere Sammlung besitzt ein sehr merkwürdiges, noch von Soemmerring ${ }^{1}$ ) stammendes Präparat, welches bereits von Leuckart in seiner bekannten Abhandlung "über das Weber'sche Organ und seine Metamorphosen " beschrieben worden ist ${ }^{2}$ ). Der Penis gleicht hier noch mehr einer grossen Clitoris, von deren Präputium zwei flügelförmige kleine Schamlippen abgehen, welche ein ziemlich weites trichterförmiges Vestibulum umgeben. Unterhalb der Harnröhrenöffnung findet sich hier zwischen zwei kleinen hymenartigen Schleimhautfalten, ganz wie bei der Raab, der enge Eingang zu der schlauchförmigen, nach aufwärts erweiterten, $5-6 \mathrm{~cm}$ langen Vagina, welche nach oben blind endet. Hier inseriren sich die beiden Vasa deferentia; auch sind kleine Rudimente von Samenblasen an der Insertionsstelle bemerkbar; die Ausführungsgänge verlaufen in leistenartigen Vorsprüngen an beiden Seiten in der vorderen Wand der Scheide. Bemerkenswerth sind in der letzteren zahlreiche Querfalten, welche gegen die Mündung hin in der Mitte eine Art vorderer Columna rugarum darstellen. Die Prostata fehlt. Der eine (jetzt nur noch allein vorhandene) Hode lag hinter dem Leistenring in einer Hydrocele; er ist $3 \mathrm{~cm}$ lang, $2 \mathrm{~cm}$ breit, und $1 \mathrm{~cm}$ dick, der Nebenhode ist deutlich erkennbar.

An einem sehr ähnlichen Präparat der Sammlung von einem neugebornen Kinde ist ebenfalls ein flaschenförmiger Uterus masculinus vorhanden, welcher jedoch noch in die Harnröhre mündet ${ }^{3}$ ), an einem dritten Präparat, ebenfalls am Neugeborenen, ist der Uterus masculinus kleiner, und die übrigen Verhältnisse nähern sich mehr den normalen ${ }^{4}$ ).

In dem ersten dieser und in anderen hierhergehörigen Fällen ist es also durch Persistenz und nachträgliche Weiterentwickelung der Müller'schen Gänge zur Bildung einer Vagina gekommen,

1) Präp. I, No. 1384, Catalog des v. Soemmering'schen Museums No. 49.

2) Illustrirt. medic. Zeitung, herausgegeben von G. Rubner. Bd.I. 1852. S. 89. Fig. 18, 19.

3) Präp. No. I, 1208. cf. Leuckart, 1. c. S.87. Ahlfeld, Atlas der Missbildungen. Taf. 40. Fig. 3, 4, 5.

4) Präp. I., No. 1209. cf. Leuckart, S. 88. 
welcher der Uterus masculinus entspricht. Die Wolff'schen oder Gartner'schen Gänge (Vasa deferentia) verlaufen in der Regel eine Strecke weit in der vorderen Wand des Uterus masc., um sich dann entweder in demselben, oder neben ihm am Colliculus seminalis zu öffnen.

Unser Fall ist nun aber noch ausgezeichnet durch das Vorhandensein eines ziemlich stark entwickelten fleischigen Uterus, welcher sich wahrscheinlich nach oben in Hörner oder vielleicht sogar Tuben fortsetzt. Ueber das nähere Verhalten derselben vermochten wir indess durch die Untersuchung keinen Aufschluss zu erhalten. Wir können nur aus der Analogie mit anderen Fällen schliessen, dass die Tuben, wenn auch wahrscheinlich nur rudimentär, so doch vorhanden sind; vollständig werden sie wohl in keinem Falle vermisst, in welchem es zur Ausbildung eines fleischigen Uteruskörpers gekommen ist. Wir verweisen in dieser Beziehung auf die übersichtliche Zusammenstellung von Abbildungen derartiger Fälle in dem Ahlfeld'schen Atlas der Missbildungen.

In welcher Weise sich die Vasa deferentia bei der Raab mit dem Uterus vereinigen, ob Rudimente von. Samenblasen vorhanden sind, oder nicht, endlich, was die Hauptsache ist, wie sich die Geschlechtsdrüsen verhalten, diese Fragen können ohne Autopsie nicht sicher entschieden werden. Was die ersteren betrifft, so sei hier nur bemerkt, dass bei vollständigem Uterus masc. die Wolff'schen oder Gartner'schen Gänge in der Regel schon an den Körper des Uterus treten, mit welchem sie Anfangs durch das Bindegewebe der Ligam. lata vereinigt sind, um sich dann am Uebergang in die Scheide in die vordere Wand derselben einzusenken, so z. B. in den Fällen von Me yer-Klebs (wahrscheinlich Hermaphr. lateralis), von Mayer und Anderen. Bekanntlich lässt sich auch am normalen weiblichen Uterus beim. Fötus ein analoger Verlauf jener Gänge nachweisen [Beigel ${ }^{\mathrm{T}}$ )]. Ganz ähnliche Verhältnisse finden wir in den androgynen Genitalien von Wiederkäuern, besonders Ziegen, von welchem unsere Sammlung eine ganze Reihe sehr instructiver Präparate besitzt.

Alles in Allem glaube ich mit grosser Wahrscheinlichkeit annehmen zu dürfen, dass die "Marie Raab" thatsächlich männ-

1) cf. Kölliker, Entwickelungsgeschichte. S.987. 
lichen Geschlechtes ist, und einen hohen Grad von Hermaphroditismus spurius der inneren und äusseren Genitalien mit allgemeinem weiblichem Habitus darbietet, dass aber die Möglichkeit eines Hermaphroditismus verus lateralis bei ihr nicht ausgeschlossen ist.

\section{Erklärung der Abbildungen.}

Tafel IX.

Fig. 1. Die äusseren Genitalien der Marie Raab, in natürlieher Grösse von vorn und etwas von rechts gesehen.

Fig. 2. Die Genitalien von vorn gesehen, mit etwas in die Höhe gehobenem Penis und auseinandergezogenen grossen Labien, so dass das Vestibulum etwas erweitert erscheint, a Glans penis, b Präputium, c das doppelte Frenulum. d Flache kahnförmige Vertiefung zwischen den beiden Hautfalten e, welche den kleinen Labien entsprechen, und nach abwärts durch eine Raphe f vereinigt sind. g Grosse Schamlippen, h Frenulum derselben, i Vestibulum, k Mündung der Harnröhre, 1 die beiden kleinen Hymen-artigen Hautfalten, zwischen welchen die Mündung der Vagina verborgen ist.

Fig. 3. Muthmaassliches Verhalten der inneren Genitalien der Raab. $\mathrm{s}$ Symphyse. v Harnblase, $\mathrm{p}$ Penis, $1 \mathrm{~m}$ Hautfalte, welche den Labia minora entspricht, u Mündung der Harnröhre, vg and $\mathrm{vg}^{\prime}$ Oeffnung und Körper der Scheide, a Anus, ut Uterus. vd und vd' Rechtes und linkes Vas deferens mit seinem muthmaasslichen Verlauf am Uterus, $t$ t' rechte und linke Tube (zweifelhaft), te Rechter Hode. 